\title{
Tableau Calculi for the Logics of Finite $k$-Ary Trees
}

\author{
Mauro Ferrari ${ }^{1}$, Camillo Fiorentini ${ }^{1}$, and Guido Fiorino ${ }^{2}$ \\ 1 Dipartimento di Scienze dell'Informazione, Università degli Studi di Milano \\ Via Comelico, 39, 20135 Milano, Italy \\ 2 CRII, Università dell'Insubria,Via Ravasi 2, 21100 Varese, Italy
}

\begin{abstract}
We present tableau calculi for the logics $\mathbf{D}_{k}(k \geq 2)$ semantically characterized by the classes of Kripke models built on finite $k$-ary trees. Our tableau calculi use the signs $\mathbf{T}$ and $\mathbf{F}$, some tableau rules for Intuitionistic Logic and two rules formulated in a hypertableau fashion. We prove the Soundness and Completeness Theorems for our calculi. Finally, we use them to prove the main properties of the logics $\mathbf{D}_{k}$, in particular their constructivity and their decidability.
\end{abstract}

\section{Introduction}

In recent years there has been a growing interest (see [1346.77810]11) in prooftheoretical characterization of propositional intermediate logics, that is logics laying between Intuitionistic and Classical Logic. This interest is motivated by the applications of some of these logics. As an example we recall DummettGödel Logic, studied for its relationship with multi-valued and fuzzy logics 14; Jankov Logic and here-and-there Logic, studied for their application to Logic Programming [1516].

Apart from the cases of Intuitionistic and Classical Logic, the prooftheoretical characterization of Intermediate Logics given in the literature relies on variations of the standard sequent calculi or tableau calculi. As an example, the tableau calculi for the interpolable Intermediate Logics described in 110, 11 use new signs besides the usual signs $\mathbf{T}$ and $\mathbf{F}$ (we remark that the calculi of [10,11] give rise to space-efficient decision procedures). However, this approach seems hard to apply to several families of interesting Intermediate Logics. Another approach relies on hypersequent calculi, a natural generalization of sequent calculi; e.g., in [3] a hypersequent characterization of Dummett-Gödel Logic is presented, while in [7] the authors extend this approach to some families of Intermediate Logics with bounded Kripke models. However, also the approach based on hypersequent calculi or hypertableau calculi (the dualized version of hypersequents presented in [6]) seems to be inadequate to treat some Intermediate Logics and further variations are needed. An example is given in [6], where the notion of path-hypertableau calculus is introduced to treat the intermediate logic of finite-depth Kripke models.

Despite the wide research in this field, we remark that all the intermediate logics studied in the above mentioned papers fail to be constructive, where 
we call constructive any intermediate logic $\mathbf{L}$ satisfying the disjunction property: $A \vee B \in \mathbf{L}$ implies $A \in \mathbf{L}$ or $B \in \mathbf{L}$. As it is well-known, there exists a continuum of constructive intermediate logics 59], but, as far as we know, no proof-theoretical characterizations of constructive logics are known, apart from those given in [2. In that paper generalized tableau calculi for the constructive $\operatorname{logics} \mathbf{D}_{k}(k \geq 2)$ and for the constructive Kreisel-Putnam Logic are presented; however, such calculi are far from being genuine tableau calculi and are highly inefficient. Indeed, they are obtained by adding to the intuitionistic tableau calculus a special rule allowing us to introduce, at any point of the derivation, a suitable $\mathbf{T}$-signed instance of the schema characterizing the logic.

In this paper we provide tableau calculi for the intermediate constructive $\operatorname{logics} \mathbf{D}_{k}(k \geq 2)$ of finite $k$-ary trees. $\mathbf{D}_{k}$ is the set of all the formulas valid in every Kripke model built on a finite $k$-ary tree. These logics have been introduced in [13, where a finite axiomatization of every $\mathbf{D}_{k}$ is given, and their decidability is proved. Our proof-theoretical characterization is based on a $h y$ brid tableau calculus that uses the two usual signs $\mathbf{T}$ and $\mathbf{F}$, some tableau rules for Intuitionistic Logic and two rules formulated in a hypertableau fashion (a structural rule and a purely logical rule). Then we use such calculi to provide a proof of the main properties of the logics $\mathbf{D}_{k}$, in particular their constructivity and their decidability.

The paper is organized as follows: in Section 2 we introduce the logics $\mathbf{D}_{k}$ providing both the axiomatization and the semantical characterization in terms of families of Kripke models. In Section 3 we introduce the calculi $\mathbf{T D}_{k}$ and we prove that they characterize the logics $\mathbf{D}_{k}$. Finally, in Section 4 we use these calculi to prove the main properties of the $\operatorname{logics} \mathbf{D}_{k}$.

\section{Preliminaries}

Here we consider the propositional language based on a denumerable set of atomic symbols and the logical constants $\perp, \wedge, \vee, \rightarrow$. We denote with $p, q, \ldots$, possibly with indexes, the atomic symbols and with $A, B, \ldots$, possibly with indexes, arbitrary formulas. Moreover, as usual in the setting of intermediate logics, $\neg A$ is defined as $A \rightarrow \perp$. Int and $\mathbf{C l}$ denote respectively the set of intuitionistically and classically valid formulas.

An intermediate propositional logic (see, e.g., [5]) is any set L of formulas satisfying the following conditions: (i) L is consistent; (ii) Int $\subseteq$ L; (iii) L is closed under modus ponens; (iv) L is closed under propositional substitution (where a propositional substitution is any function mapping every propositional variable to a formula). It is well-known that, for any intermediate logic $\mathrm{L}, \mathrm{L} \subseteq \mathbf{C l}$.

Many intermediate logics can be semantically characterized by families of Kripke models. A (propositional) Kripke model (see, e.g., [5]) is a structure $\underline{K}=\langle P, \leq, \Vdash\rangle$, where $\langle P, \leq\rangle$ is a poset (partially ordered set), and $\Vdash$ (the forcing relation) is a binary relation between elements of $P$ and atomic symbols such that, for every atomic symbol $p, \alpha \Vdash p$ implies $\beta \Vdash p$ for every $\beta \in P$ such that $\alpha \leq \beta$. The forcing relation is extended to arbitrary formulas as follows: 
1. $\alpha \nVdash \perp$;

2. $\alpha \Vdash B \wedge C$ iff $\alpha \Vdash B$ and $\alpha \Vdash C$;

3. $\alpha \Vdash B \vee C$ iff $\alpha \Vdash B$ or $\alpha \Vdash C$;

4. $\alpha \Vdash B \rightarrow C$ iff, for every $\beta \in P$ such that $\alpha \leq \beta, \beta \Vdash B$ implies $\beta \Vdash C$.

We write $\alpha \nVdash A$ to mean that $\alpha \Vdash A$ does not hold. We remark that, according to the above interpretation, $\alpha \Vdash \neg A$ iff, for every $\beta \in P$ such that $\alpha \leq \beta$, we have $\beta \nVdash A$.

It is easy to check that the forcing relation meets the monotonicity condition:

Proposition 1. For every formula $A$, Kripke model $\underline{K}=\langle P, \leq, \Vdash\rangle$ and element $\alpha$ in $\underline{K}$, if $\alpha \Vdash A$ then $\beta \Vdash A$ for every $\beta \in P$ such that $\alpha \leq \beta$.

Given a Kripke model $\underline{K}=\langle P, \leq, \Vdash\rangle$, we write $\alpha<\beta$ to mean that $\alpha \leq \beta$ and $\alpha \neq \beta$. Given $\alpha \in P$, we call immediate successor of $\alpha$ (in $\underline{K}$ ) any $\beta \in P$ such that $\alpha<\beta$ and, for every $\gamma \in P$, if $\alpha \leq \gamma \leq \beta$ then either $\gamma=\alpha$ or $\gamma=\beta$. We call final element of $\underline{K}$ any $\phi \in P$ such that, for every $\alpha \in P$, if $\phi \leq \alpha$ then $\phi=\alpha$. It is easy to check that a final element $\phi$ of $\underline{K}$ behaves like a classical interpretation, that is, for every formula $A$, either $\phi \Vdash A$ or $\phi \Vdash \neg A$.

A formula $A$ is valid in a Kripke model $\underline{K}$ if $\alpha \Vdash A$ for all $\alpha \in P$. If $\mathcal{K}$ is a non empty class of Kripke models, $A$ is valid in $\mathcal{K}$ if it is valid in every model of $\mathcal{K}$.

For every $k \geq 2$, let $\left(D_{k}\right)$ be the axiom schema

$$
\bigwedge_{i=0}^{k}\left(\left(p_{i} \rightarrow \bigvee_{j \neq i} p_{j}\right) \rightarrow \bigvee_{j \neq i} p_{j}\right) \rightarrow \bigvee_{i=0}^{k} p_{i}
$$

and let $\mathbf{D}_{k}$ denote the closure under modus ponens and propositional substitution of the set containing Int and all the instances of the axiom schema $\left(D_{k}\right)$. As shown in [13] every $\mathbf{D}_{k}(k \geq 2)$ is an intermediate logic and the sequence $\left\{\mathbf{D}_{k}\right\}_{k \geq 2}$ has the following properties:

$-\cap_{k \geq 2} \mathbf{D}_{k}=\mathbf{I n t}$

- For every $k \geq 2, \mathbf{D}_{k} \supset \mathbf{D}_{k+1}$;

- For every $k \geq 2, \mathbf{D}_{k}$ has the disjunction property: that is, for every formula of the kind $A \vee B$, if $A \vee B \in \mathbf{D}_{k}$, then $A \in \mathbf{D}_{k}$ or $B \in \mathbf{D}_{k}$;

- Every $D_{k}$ is decidable.

Now, let $\mathcal{T}_{k}$ be the the family of all the Kripke models $\underline{K}=\langle P, \leq, \Vdash\rangle$ where:

$-\langle P, \leq\rangle$ is a finite tree;

- Given $\alpha \in P, \alpha$ has at most $k$ immediate successors in $\langle P, \leq\rangle$.

In [13] the following result is proved:

Theorem 1. For every $k \geq 2, A \in \mathbf{D}_{k}$ iff $A$ is valid in $\mathcal{T}_{k}$.

Hence the above result shows that every logic $\mathbf{D}_{k}$ is characterized by the class of finite $k$-ary trees. 
The above quoted properties of the logics $\mathbf{D}_{k}$ are proved in [13] by means of semantical tools. In particular the decidability of $\mathbf{D}_{k}$ relies on the decidability of the second order theory describing the validity of formulas in $\mathcal{T}_{k}$ and the disjunction property follows from a property of the class of models $\mathcal{T}_{k}$. In the following sections we introduce a tableau calculus for every $\mathbf{T D}_{k}$ and then we use the properties of such a calculus to deduce the decidability and the disjunction property for $\mathbf{D}_{k}$.

\section{The Sequence of Tableau Calculi $\mathrm{TD}_{k}(k \geq 2)$}

A signed formula (swff for short) is an expression of the form $\mathbf{T} X$ or $\mathbf{F} X$ where $X$ is any formula. The meaning of the signs $\mathbf{T}$ and $\mathbf{F}$ is as follows: given a Kripke model $\underline{K}=\langle P, \leq, \Vdash\rangle$ and a swff $H, \alpha \in P$ realizes $H$ (in symbols $\alpha \triangleright H$ ) if $H \equiv \mathbf{T} X$ and $\alpha \Vdash X$, or $H \equiv \mathbf{F} X$ and $\alpha \nVdash X$. $\alpha \not H$ means that $\alpha \triangleright H$ does not hold. $\alpha$ realizes a set of swff's $S(\alpha \triangleright S)$ if $\alpha$ realizes every swff in $S$. By Proposition 1, if $\alpha \triangleright \mathbf{T} X$ then $\beta \triangleright \mathbf{T} X$ for every $\beta \in P$ such that $\alpha \leq \beta$. On the other hand, if $\alpha \triangleright \mathbf{F} X$, then can exist $\beta \in P$ such that $\alpha \leq \beta$ and $\beta \not \mathbf{F} X$.

A hyperset is an expression of the form

$$
S_{1}|\ldots| S_{n}
$$

where, for all $i=1, \ldots, n, S_{i}$ is a set of swff's. $S_{i}$ is called a component of the hyperset. We call simple hyperset a hyperset containing exactly one component. A configuration is an expression of the form

$$
\Psi_{1}\|\ldots\| \Psi_{m}
$$

where, for all $i=1, \ldots, m, \Psi_{i}$ is a hyperset. $\Psi_{i}$ is called a component of the configuration. A simple configuration is a configuration where every component is a simple hyperset.

The intended meaning of the symbol $\mid$ is conjunctive while the one of the symbol $\|$ is disjunctive. Formally, given a Kripke model $\underline{K}=\langle P, \leq, \Vdash\rangle, \underline{K}$ realizes a hyperset $S_{1}|\ldots| S_{n}$ if, for every $i=1, \ldots, n$, there exists $\alpha_{i} \in \bar{P}$ such that $\alpha_{i} \triangleright S_{i}$. On the other hand, $\underline{K}$ realizes a configuration $\Psi_{1}\|\ldots\| \Psi_{m}$ if there exists a hyperset $\Psi_{j}$, with $j \in\{1, \ldots, m\}$, such that $\underline{K}$ realizes $\Psi_{j}$.

The rules of Table 1 are common to all the calculi $\mathbf{T D}_{k}$ for $k \geq 2$ and are independent of the parameter $k$. The rule properly characterizing the tableau calculus $\mathbf{T} \mathbf{D}_{k}$ is $\mathrm{D}_{k}$ and it will be introduced in a while. Before that we introduce some notations. First of all, in the rules of the calculus $\mathbf{T D}_{k}$, we simply denote with $S, H_{1}, \ldots, H_{h}$ the set $S \cup\left\{H_{1}, \ldots, H_{h}\right\}$. The rules apply to configurations but, to simplify the notation, we omit the components of the configuration not involved in the rule. E.g., the schema

$$
\frac{S_{1}\|\ldots\| S, \mathbf{T}(A \wedge B)\|\ldots\| S_{n}}{S_{1}\|\ldots\| S, \mathbf{T} A, \mathbf{T} B\|\ldots\| S_{n}} \mathbf{T} \wedge
$$

illustrates an application of the $\mathbf{T} \wedge$-rule. In every rule we distinguish two parts: the premise, that is the configuration above the line, and the conclusion, that is the configuration below the line. We remark that all the rules of Table 1 but 
Table 1. Rules common to all the $\mathbf{T D}_{k}$

$\frac{S, \mathbf{T}(A \wedge B)}{S, \mathbf{T} A, \mathbf{T} B} \mathbf{T} \wedge \frac{S, \mathbf{F}(A \wedge B)}{S, \mathbf{F} A \| S, \mathbf{F} B} \mathbf{F} \wedge$
$\frac{S, \mathbf{T}(A \vee B)}{S, \mathbf{T} A \| S, \mathbf{T} B} \mathbf{T} \vee \frac{S, \mathbf{F}(A \vee B)}{S, \mathbf{F} A} \mathbf{F} \vee_{1} \frac{S, \mathbf{F}(A \vee B)}{S, \mathbf{F} B} \mathbf{F} \vee_{2}$
$\frac{S, \mathbf{T} A, \mathbf{T}(A \rightarrow B)}{S, \mathbf{T} A, \mathbf{T} B} \mathbf{T} \rightarrow A$ tom with $A$ an atom
$\frac{S, \mathbf{T}((A \wedge B) \rightarrow C)}{S, \mathbf{T}(A \rightarrow(B \rightarrow C))} \mathbf{T} \rightarrow \wedge \frac{S, \mathbf{T}((A \vee B) \rightarrow C)}{S, \mathbf{T}(A \rightarrow C), \mathbf{T}(B \rightarrow C)} \mathbf{T} \rightarrow \vee$
$\frac{S, \mathbf{T}((A \rightarrow B) \rightarrow C)}{S, \mathbf{F}(A \rightarrow B), \mathbf{T}((A \rightarrow B) \rightarrow C) \| S, \mathbf{T} C} \mathbf{T} \rightarrow \rightarrow$
$\frac{S_{1}|\ldots| S_{i}|\ldots| S_{n}}{S_{i}}$ Weak

Weak only involve simple configurations (both the premise and the consequence of such rules are simple configurations). On the other hand, Weak has a non simple configuration as premise and a simple configuration as consequence; on the contrary, as we will see, the rule $\mathrm{D}_{k}$ properly characterizing $\mathbf{T} \mathbf{D}_{k}$ has a simple configuration as premise and (in general) a non simple configuration as consequence.

We call main set of swff's of a rule the set of swff's that are in evidence in the premise of the rule; when the main set of swff's of a rule contains just a swff we call it the main swff of the rule. As an example, $\mathbf{T}(A \wedge B)$ is the main swff of the rule $\mathbf{T} \wedge$ while $\{\mathbf{T} A, \mathbf{T}(A \rightarrow B)\}$ is the main set of swff's of the rule $\mathbf{T} \rightarrow$ Atom. The rule Weak is a structural rule; it acts on components of a hyperset and it does not have a main set of swff's.

The rule properly characterizing the calculus $\mathbf{T} \mathbf{D}_{k}$ is $\mathrm{D}_{k}$ that applies to the premise

$$
S, \mathbf{F}\left(A_{1} \rightarrow B_{1}\right), \ldots, \mathbf{F}\left(A_{n} \rightarrow B_{n}\right), \mathbf{T}\left(\left(C_{1} \rightarrow D_{1}\right) \rightarrow E_{1}\right), \ldots, \mathbf{T}\left(\left(C_{m} \rightarrow D_{m}\right) \rightarrow E_{m}\right)
$$

Let

$$
\begin{aligned}
& U=\left\{\mathbf{F}\left(A_{1} \rightarrow B_{1}\right), \ldots, \mathbf{F}\left(A_{n} \rightarrow B_{n}\right)\right\} \\
& V=\left\{\mathbf{T}\left(\left(C_{1} \rightarrow D_{1}\right) \rightarrow E_{1}\right), \ldots, \mathbf{T}\left(\left(C_{m} \rightarrow D_{m}\right) \rightarrow E_{m}\right)\right\}
\end{aligned}
$$

and let $U \cup V$ be the main set of swff's of the rule. Now, let $\Sigma_{U}$ be the set containing all the subsets of $U$ different from $U$ itself and the empty set. We remark that the cardinality of $\Sigma_{U}$ is $2^{n}-2$. We denote with $\Sigma_{U}^{k}$ the set of all the complete $k$-sequences of $\Sigma_{U}$, that is the set of all the sequences $\Phi_{1}, \ldots, \Phi_{h}$ of elements of $\Sigma_{U}$ such that: 
$-h \leq k$

$-\bigcup_{1 \leq i \leq h} \Phi_{i}=U$

We associate with every sequence $\sigma \equiv \Phi_{1}, \ldots, \Phi_{h}$ belonging to $\Sigma_{U}^{k}$ the hyperset

$$
\mathcal{H}_{\sigma} \equiv S_{c}, \Phi_{1}, \Psi_{1}|\ldots| S_{c}, \Phi_{h}, \Psi_{h}
$$

where, $S_{c}=\{\mathbf{T} A \mid \mathbf{T} A \in S\}$ and, for $i=1, \ldots, h$

$$
\begin{aligned}
\Psi_{i}= & \left\{\mathbf{T} E \mid \mathbf{T}((C \rightarrow D) \rightarrow E) \in V \text { and } \mathbf{F}(C \rightarrow D) \notin \Phi_{i}\right\} \\
& \cup\left\{\mathbf{T}((C \rightarrow D) \rightarrow E) \mid \mathbf{T}((C \rightarrow D) \rightarrow E) \in V \text { and } \mathbf{F}(C \rightarrow D) \in \Phi_{i}\right\} \\
& \cup\{\mathbf{T}((C \rightarrow D) \rightarrow E) \mid \mathbf{T}((C \rightarrow D) \rightarrow E) \in V \text { and } \mathbf{F}(C \rightarrow D) \notin U\}
\end{aligned}
$$

The rule $\mathrm{D}_{k}$ is

$$
\frac{S, \mathbf{F}\left(A_{1} \rightarrow B_{1}\right), \ldots, \mathbf{F}\left(A_{n} \rightarrow B_{n}\right), \mathbf{T}\left(\left(C_{1} \rightarrow D_{1}\right) \rightarrow E_{1}\right), \ldots, \mathbf{T}\left(\left(C_{m} \rightarrow D_{m}\right) \rightarrow E_{m}\right)}{S_{c}, \Gamma_{1}, \Delta_{1}\|\ldots\| S_{c}, \Gamma_{n}, \Delta_{n}\left\|\mathcal{H}_{\sigma_{1}}\right\| \ldots \| \mathcal{H}_{\sigma_{r}}} \mathrm{D}_{k}
$$

where $\sigma_{1}, \ldots, \sigma_{r}$ are all the complete $k$-sequences in $\Sigma_{U}^{k}$ and, for $i=1, \ldots, n$

$$
\begin{aligned}
\Gamma_{i}= & U \backslash\left\{\mathbf{F}\left(A_{i} \rightarrow B_{i}\right)\right\} \cup\left\{\mathbf{T} A_{i}, \mathbf{F} B_{i}\right\} \\
\Delta_{i}= & \left\{\mathbf{T}\left(B_{i} \rightarrow E\right) \mid \mathbf{T}\left(\left(A_{i} \rightarrow B_{i}\right) \rightarrow E\right) \in V\right\} \\
& \cup\left\{\mathbf{T}((C \rightarrow D) \rightarrow E) \in V \mid C \rightarrow D \not \equiv A_{i} \rightarrow B_{i}\right\}
\end{aligned}
$$

The following is an example of application of $\mathrm{D}_{2}$ where the components of the consequence occur in different lines

$$
\begin{gathered}
\mathbf{F}(A \rightarrow B), \mathbf{F}(C \rightarrow D), \mathbf{F}(E \rightarrow G), \mathbf{T}((A \rightarrow B) \rightarrow H) \\
\mathbf{T} A, \mathbf{F} B, \mathbf{F}(C \rightarrow D), \mathbf{F}(E \rightarrow G), \mathbf{T}(B \rightarrow H) \| \\
\mathbf{F}(A \rightarrow B), \mathbf{T} C, \mathbf{F} D, \mathbf{F}(E \rightarrow G), \mathbf{T}((A \rightarrow B) \rightarrow H) \| \\
\mathbf{F}(A \rightarrow B), \mathbf{F}(C \rightarrow D), \mathbf{T} E, \mathbf{F} G, \mathbf{T}((A \rightarrow B) \rightarrow H) \| \\
\mathbf{F}(A \rightarrow B), \mathbf{T}((A \rightarrow B) \rightarrow H) \mid \mathbf{F}(C \rightarrow D), \mathbf{F}(E \rightarrow G), \mathbf{T} H \| \\
\mathbf{F}(C \rightarrow D), \mathbf{T} H \mid \mathbf{F}(A \rightarrow B), \mathbf{F}(E \rightarrow G), \mathbf{T}((A \rightarrow B) \rightarrow H) \| \\
\mathbf{F}(E \rightarrow G), \mathbf{T} H \mid \mathbf{F}(A \rightarrow B), \mathbf{F}(C \rightarrow D), \mathbf{T}((A \rightarrow B) \rightarrow H) \| \\
\mathrm{D}_{2} \\
\mathbf{F}(A \rightarrow B), \mathbf{F}(C \rightarrow D), \mathbf{T}((A \rightarrow B) \rightarrow H) \mid \mathbf{F}(A \rightarrow B), \mathbf{F}(E \rightarrow G), \mathbf{T}((A \rightarrow B) \rightarrow H) \| \\
\mathbf{F}(A \rightarrow B), \mathbf{F}(C \rightarrow D), \mathbf{T}((A \rightarrow B) \rightarrow H) \mid \mathbf{F}(C \rightarrow D), \mathbf{F}(E \rightarrow G), \mathbf{T} H \| \\
\mathbf{F}(A \rightarrow B), \mathbf{F}(E \rightarrow G), \mathbf{T}((A \rightarrow B) \rightarrow H) \mid \mathbf{F}(C \rightarrow D), \mathbf{F}(E \rightarrow G), \mathbf{T} H
\end{gathered}
$$

We remark that, if $U=\left\{\mathbf{F}\left(A_{1} \rightarrow B_{1}\right)\right\}$ the corresponding instance of $\mathrm{D}_{k}$ is

$$
\frac{S, \mathbf{F}\left(A_{1} \rightarrow B_{1}\right), \mathbf{T}\left(\left(C_{1} \rightarrow D_{1}\right) \rightarrow E_{1}\right), \ldots, \mathbf{T}\left(\left(C_{m} \rightarrow D_{m}\right) \rightarrow E_{m}\right)}{S_{c}, \mathbf{T} A_{1}, \mathbf{F} B_{1}, \Delta_{1}} \mathrm{D}_{k}
$$

which is a purely intuitionistic rule.

A set $S$ of swff's is contradictory if one of the following conditions holds:

1. $\mathbf{T} A \in S$ and $\mathbf{F} A \in S$;

2. $\mathbf{T} \perp \in S$.

A hyperset $S_{1}|\ldots| S_{n}$ is contradictory if at least one of the $S_{i}$ is contradictory and a configuration $\Psi_{1}\|\ldots\| \Psi_{m}$ is contradictory if all the $\Psi_{i}$ are contradictory. It is easy to check that: 
Proposition 2. If a configuration is contradictory then it is not realizable in any Kripke model.

Given the tableau calculus $\mathbf{T D}_{k}$, a $\mathbf{T D}_{k}$-proof table for a configuration $\mathcal{K}_{1}$ is a finite sequence of configurations $\mathcal{K}_{1}, \ldots, \mathcal{K}_{n}$, where the configuration $\mathcal{K}_{i+1}$ is obtained from $\mathcal{K}_{i}=\Psi_{1}\|\ldots\| \Psi_{m}$ by applying a rule to a non-contradictory hyperset. A closed $\mathbf{T D}_{k}$-proof table is a $\mathbf{T} \mathbf{D}_{k}$-proof table $\mathcal{K}_{1}, \ldots, \mathcal{K}_{n}$ where the last configuration is contradictory. Closed $\mathbf{T} \mathbf{D}_{k}$-proof tables are the proofs of our calculus $\mathbf{T D}_{k}$. A formula $A$ is provable in $\mathbf{T} \mathbf{D}_{k}$ if there exists a closed $\mathbf{T} \mathbf{D}_{k^{-}}$ proof table for the configuration $\{\mathbf{F} A\}$ (the configuration consisting of the set $\{\mathbf{F} A\}$ only).

Now, our aim is to prove that, for every $k \geq 2$, the calculus $\mathbf{T D}_{k}$ is sound and complete with respect to the class of Kripke models $\mathcal{T}_{k}$. As usual the main step of the Soundness Theorem consists in proving that the rules of the calculus preserve realizability.

Lemma 1. If the premise of a rule of $\mathbf{T D}_{k}$ is realized in a model $\underline{K} \in \mathcal{T}_{k}$, then the consequence of the rule is realized in $\underline{K}$.

Proof. We only analyze the case of the rule $\mathrm{D}_{k}$ the other cases being trivial. So, let us assume that $\underline{K}=\langle P, \leq, \Vdash\rangle \in \mathcal{T}_{k}$ and that an element $\alpha \in P$ realizes the set of swff's $\Gamma=S \cup U \cup V$, where $S$ is any set of swff's and

$$
\begin{aligned}
U & =\left\{\mathbf{F}\left(A_{1} \rightarrow B_{1}\right), \ldots, \mathbf{F}\left(A_{n} \rightarrow B_{n}\right)\right\} \\
V & =\left\{\mathbf{T}\left(\left(C_{1} \rightarrow D_{1}\right) \rightarrow E_{1}\right), \ldots, \mathbf{T}\left(\left(C_{m} \rightarrow D_{m}\right) \rightarrow E_{m}\right)\right\}
\end{aligned}
$$

Now, since $\underline{K}$ is finite, there exists an element $\beta$ such that $\alpha \leq \beta, \beta \triangleright U$ and, for every $\gamma>\beta, \gamma \not U$. We have two cases:

Case 1: There exists an $i \in\{1, \ldots, n\}$ such that $\beta \triangleright\left\{\mathbf{T} A_{i}, \mathbf{F} B_{i}\right\}$. This implies that $\beta \triangleright \Gamma_{i}$ where $\Gamma_{i}=U \backslash\left\{\mathbf{F}\left(A_{i} \rightarrow B_{i}\right)\right\} \cup\left\{\mathbf{T} A_{i}, \mathbf{F} B_{i}\right\}$; moreover, since $\alpha \leq \beta$, $\beta \triangleright S_{c} \cup V$ and, since $\beta \triangleright \mathbf{T}\left(\left(A_{i} \rightarrow B_{i}\right) \rightarrow E\right)$ implies $\beta \triangleright \mathbf{T}\left(B_{i} \rightarrow E\right)$, we get $\beta \triangleright S_{c}, \Gamma_{i}, \Delta_{i}$.

Case 2: For every $i \in\{1, \ldots, n\}, \beta \not\left\{\mathbf{T} A_{i}, \mathbf{F} B_{i}\right\}$. Then, for every $\mathbf{F}\left(A_{i} \rightarrow\right.$ $\left.B_{i}\right) \in U$ there exists $\gamma \in P$ such that $\beta<\gamma$ and $\gamma \triangleright\left\{\mathbf{T} A_{i}, \mathbf{F} B_{i}\right\}$. Let $\Theta$ be the set of all $\gamma \in P$ such that $\beta<\gamma$ and $\gamma \triangleright\left\{\mathbf{T} A_{i}, \mathbf{F} B_{i}\right\}$ for some $\mathbf{F}\left(A_{i} \rightarrow B_{i}\right) \in U$. Since $\underline{K} \in \mathcal{T}_{k}, \beta$ has at most $k$ immediate successors. Let $\delta_{1}, \ldots, \delta_{h}(h \leq k)$ be the distinct immediate successors of $\beta$ such that there exists $\gamma \in \Theta$ such that $\delta_{i} \leq \gamma$. Let, for $i=1, \ldots, h$

$$
\begin{aligned}
\Phi_{i}= & \left\{\mathbf{F}(A \rightarrow B) \in U \mid \delta_{i} \triangleright \mathbf{F}(A \rightarrow B)\right\} \\
\Psi_{i}= & \left\{\mathbf{T} E \mid \mathbf{T}((C \rightarrow D) \rightarrow E) \in V \text { and } \mathbf{F}(C \rightarrow D) \notin \Phi_{i}\right\} \\
& \cup\left\{\mathbf{T}((C \rightarrow D) \rightarrow E) \mid \mathbf{T}((C \rightarrow D) \rightarrow E) \in V \text { and } \mathbf{F}(C \rightarrow D) \in \Phi_{i}\right\} \\
& \cup\{\mathbf{T}((C \rightarrow D) \rightarrow E) \mid \mathbf{T}((C \rightarrow D) \rightarrow E) \in V \text { and } \mathbf{F}(C \rightarrow D) \notin U\}
\end{aligned}
$$

Since $\cup_{i=1}^{h} \Phi_{i}=U, \sigma=\Phi_{1}, \ldots, \Phi_{h}$ is a complete $k$-sequence of $\Sigma_{U}^{k}$. Clearly $\delta_{i} \triangleright \Phi_{i}$; moreover, since $\delta_{i} \triangleright V$ and, for all $\mathbf{F}(C \rightarrow D) \notin \Phi_{i}, \delta_{i} \Vdash C \rightarrow D$, it holds that $\delta_{i} \triangleright \Psi_{i}$. Therefore, $\delta_{i} \triangleright S_{c}, \Phi_{i}, \Psi_{i}$ for all $i \in\{1, \ldots, h\}$ and the hyperset $\mathcal{H}_{\sigma}$ is realized in $\underline{K}$. Thus, both in Case 1 and in Case $2, \beta$ realizes the conclusion of $\mathrm{D}_{k}$. 
From the above lemma we deduce that, if $A$ is not valid in $\mathcal{T}_{k}$, then no closed $\mathbf{T D}_{k}$-proof table for $\{\mathbf{F} A\}$ can exist. Indeed, let $\underline{K}=\langle P, \leq, \Vdash\rangle$ be a model of $\mathcal{T}_{k}$ such that $A$ is not valid in $\underline{K}$ and let us assume that there exists a closed $\mathbf{T D}_{k}$-proof table $\tau$ for $\{\mathbf{F} A\}$. Since $\underline{K}$ realizes $\{\mathbf{F} A\}$, by the previous lemma, $\underline{K}$ realizes the last configuration of $\tau$ against Proposition 2 . It follows that $A$ is not provable in $\mathbf{T D}_{k}$, hence:

Theorem 2 (Soundness). If $A$ is provable in $\mathbf{T D}_{k}$, then $A$ is valid in $\mathcal{T}_{k}$.

Now, a finite set $S$ of swff's is $\mathbf{T D}_{k}$-consistent if no $\mathbf{T D}_{k}$-proof table starting from $S$ is closed. To prove the completeness of $\mathbf{T} \mathbf{D}_{k}$ we provide a procedure that, given a finite and $\mathbf{T D}_{k}$-consistent set $S$ of swff's, allows us to build a Kripke model $\underline{K}_{\mathbf{D}}(S)$ whose root realizes $S$. Our technique is similar to the one used in [1], which is an adaptation of Fitting's one described in 12. The construction consists of two steps. In the first step we construct the sequence $\left\{S_{i}\right\}_{i \in \omega}$ of sets of swff's and the set of swff's $\bar{S}$, called the node set of $S$. $\bar{S}$ will be the root of the model $\underline{K}_{\mathbf{D}}(S)$ and its forcing relation is determined by the signed atoms belonging to $\bar{S}$. In the second step we construct the successor sets $\Delta_{1}, \ldots, \Delta_{h}$ of $\bar{S}$. The model $\underline{K}_{\mathbf{D}}(S)$ will be constructed by iterating the two steps on $\Delta_{1}, \ldots, \Delta_{h}$.

Let us consider a finite and $\mathbf{T D}_{k}$-consistent set $S$ of swff's and let $A_{1}, \ldots, A_{n}$ be any listing of swff's of $S$ (without repetitions of swff's). Starting from this listing we construct the sequence $\left\{S_{i}\right\}_{i \in \omega}$ of sets of swff's defined as follows:

$-S_{0}=S$

- Let $S_{i}=\left\{H_{1}, \ldots, H_{u}\right\} ;$ then

$$
S_{i+1}=\bigcup_{H_{j} \in S_{i}} \mathcal{U}\left(H_{j}, i\right)
$$

where, setting $S_{j}^{\prime}=\mathcal{U}\left(H_{1}, i\right) \cup \cdots \cup \mathcal{U}\left(H_{j-1}, i\right) \bigcup\left\{H_{j}, \ldots, H_{u}\right\}, \mathcal{U}\left(H_{j}, i\right)$ is defined as follows:

$(\mathcal{N} 1)$ If $H_{j} \equiv \mathbf{T}(A \wedge B)$, then $\mathcal{U}\left(H_{j}, i\right)=\{\mathbf{T} A, \mathbf{T} B\}$.

$(\mathcal{N} 2)$ If $H_{j} \equiv \mathbf{F}(A \wedge B)$, then $\mathcal{U}\left(H_{j}, i\right)=\{\mathbf{F} A\}$ if $\left(S_{j}^{\prime} \backslash\left\{H_{j}\right\}\right) \bigcup\{\mathbf{F} A\}$ is $\mathbf{T D}_{k^{-}}$ consistent and $\mathcal{U}\left(H_{j}, i\right)=\{\mathbf{F} B\}$ otherwise.

$(\mathcal{N} 3)$ If $H_{j} \equiv \mathbf{T}(A \vee B)$, then $\mathcal{U}\left(H_{j}, i\right)=\{\mathbf{T} A\}$ if $\left(S_{j}^{\prime} \backslash\left\{H_{j}\right\}\right) \bigcup\{\mathbf{T} A\}$ is $\mathbf{T D}_{k^{-}}$ consistent and $\mathcal{U}\left(H_{j}, i\right)=\{\mathbf{T} B\}$ otherwise.

$(\mathcal{N} 4)$ If $H_{j} \equiv \mathbf{T}(A \rightarrow B)$ with $A$ an atom, then $\mathcal{U}\left(H_{j}, i\right)=\{\mathbf{T} B\}$ if $\mathbf{T} A \in S_{j}^{\prime}$, and $\mathcal{U}\left(H_{j}, i\right)=\left\{H_{j}\right\}$ otherwise.

$(\mathcal{N} 5)$ If $H_{j} \equiv \mathbf{T}((A \wedge B) \rightarrow C)$, then $\mathcal{U}\left(H_{j}, i\right)=\{\mathbf{T}(A \rightarrow(B \rightarrow C)\}$.

$(\mathcal{N} 6)$ If $H_{j} \equiv \mathbf{T}((A \vee B) \rightarrow C)$, then $\mathcal{U}\left(H_{j}, i\right)=\{\mathbf{T}(A \rightarrow C), \mathbf{T}(B \rightarrow C)\}$

$(\mathcal{N} 7)$ If $H_{j} \equiv \mathbf{T}((A \rightarrow B) \rightarrow C)$ and $\mathbf{F}(A \rightarrow B) \notin S_{j}^{\prime}$, then $\mathcal{U}\left(H_{j}, i\right)=\{\mathbf{T} C\}$ if $\left(S_{j}^{\prime} \backslash\left\{H_{j}\right\}\right) \bigcup\{\mathbf{T} C\}$ is $\mathbf{T D}_{k}$-consistent and $\mathcal{U}\left(H_{j}, i\right)=\left\{H_{j}, \mathbf{F}(A \rightarrow B)\right\}$ otherwise. If $\mathbf{F}(A \rightarrow B) \in S_{j}^{\prime}$ then $\mathcal{U}\left(H_{j}, i\right)=\left\{H_{j}\right\}$.

$(\mathcal{N} 8)$ If $H_{j}$ is a signed atom or $H_{j} \equiv \mathbf{F}(A \rightarrow B)$ or $H_{j} \equiv \mathbf{F}(A \vee B)$, then $\mathcal{U}\left(H_{j}, i\right)=\left\{H_{j}\right\}$. 
It is easy to check, by induction on $i \geq 0$, that, if $S$ is $\mathbf{T} \mathbf{D}_{k}$-consistent, then every $S_{i}$ is $\mathbf{T} \mathbf{D}_{k}$-consistent. Moreover, since $S$ is finite, every $S_{i}$ is finite and there exists an index $j$ such that $S_{i}=S_{j}$ for every $i \geq j$. Let $u$ be the first index such that $S_{u}=S_{u+1}$. We call node set of $S$ the set $\bar{S}=S_{u}$ and we call $\left\{S_{0}, \ldots, S_{u}\right\}$ the sequence generating $\bar{S}$. We remark that different listings $A_{1}, \ldots, A_{n}$ of the swff's of $S$ give rise to different sequences $\left\{S_{i}\right\}_{i \in \omega}$ and to different node sets of $S$.

The successor sets of $\bar{S}$ are defined as follows:

$(\mathcal{S} 1)$ If $\bar{S}$ contains at least one swff $H \equiv \mathbf{F}(A \vee B)$, then the only successor sets of $\bar{S}$ are $U_{1}=(\bar{S} \backslash\{H\}) \cup\{\mathbf{F} A\}$ and $U_{2}=(\bar{S} \backslash\{H\}) \cup\{\mathbf{F} B\}$. We call $U_{1}$ and $U_{2}$ the successor sets corresponding to the $\mathbf{F} \vee$-rule.

$(\mathcal{S} 2)$ If $\bar{S}$ does not contain swff's of the kind $\mathbf{F}(A \vee B)$ and contains at least one swff of the kind $\mathbf{F}(A \rightarrow B)$, then let:

$$
\begin{aligned}
& U=\{\mathbf{F}(A \rightarrow B) \mid \mathbf{F}(A \rightarrow B) \in \bar{S}\} \\
& V=\{\mathbf{T}((C \rightarrow D) \rightarrow E) \mid \mathbf{T}((C \rightarrow D) \rightarrow E) \in \bar{S}\} \\
& S=\bar{S} \backslash(U \cup V)
\end{aligned}
$$

and let

$$
S_{c}, \Gamma_{1}, \Delta_{1}\|\ldots\| S_{c}, \Gamma_{n}, \Delta_{n}\left\|\mathcal{H}_{\sigma_{1}}\right\| \ldots \| \mathcal{H}_{\sigma_{r}}
$$

be the configuration obtained by applying the rule $\mathrm{D}_{k}$ to the set $S \cup U \cup V$ where the main set of swff's is $U \cup V$. Since, by hypothesis, $\bar{S}$ is $\mathbf{T} \mathbf{D}_{k^{-}}$ consistent, at least a component of this configuration is $\mathbf{T} \mathbf{D}_{k}$-consistent, let $\Phi_{1}|\ldots| \Phi_{h}(1 \leq h \leq k)$ be such a component. By applying the Weakrule we deduce that all the $\Phi_{i}$ are $\mathbf{T} \mathbf{D}_{k}$-consistent. The only successor sets of $\bar{S}$ are $\Phi_{1}, \ldots, \Phi_{h}$. We call $\Phi_{1}, \ldots, \Phi_{h}$ the successor sets corresponding to the $D_{k}$-rule.

We remark that if $\bar{S}$ is finite and $\mathbf{T} \mathbf{D}_{k}$-consistent then every successor set of $\bar{S}$ is finite and $\mathbf{T} \mathbf{D}_{k}$-consistent; moreover $\bar{S}$ has $k$ successor sets at most.

Given a finite and $\mathbf{T} \mathbf{D}_{k}$-consistent set $S$ of swff's, we use the construction above to define the structure $\underline{K}_{\mathbf{D}}(S)=\langle P, \leq, \Vdash\rangle$ as follows:

1. $\bar{S} \in P$, where $\bar{S}$ is a node set of $S$;

2. For every $\bar{\Gamma} \in P$ and for every successor set $\Delta$ of $\bar{\Gamma}$, let $\bar{\Delta}$ be a node set of $\Delta$. Then $\bar{\Delta}$ is a member of $P$ and $\bar{\Delta}$ is an immediate successor of $\bar{\Gamma}$ in $\underline{K}_{\mathbf{D}}(S)$;

$3 . \leq$ is the transitive and reflexive closure of the immediate successor relation;

4. For every atom $p$ and for every $\bar{\Gamma} \in P, \bar{\Gamma} \Vdash p$ iff $\mathbf{T} p \in \bar{\Gamma}$.

Now, we have to prove that the structure $\underline{K}_{\mathbf{D}}(S)$ defined above is a Kripke model of $\mathcal{T}_{k}$. In particular we need to prove the finiteness of $\langle P, \leq\rangle$; to this aim we introduce the notion of degree and weight. Given a formula $A$, the degree of $A$, denoted by $d g(A)$, is defined as follows: 
- if $A \equiv p$, where $p$ is an atom, or $A \equiv \perp$, then $d g(A)=0$;

- if $A \equiv B \wedge C$ or $A \equiv B \vee C$, then $d g(A)=d g(B)+d g(C)+1$;

- if $A \equiv p \rightarrow B$ where $p$ is an atom, then $d g(A)=d g(B)+2$;

- if $A \equiv(B \wedge C) \rightarrow D$, then $d g(A)=d g(B \rightarrow(C \rightarrow D))+1$;

- if $A \equiv(B \vee C) \rightarrow D$, then $d g(A)=d g(B \rightarrow D)+d g(C \rightarrow D)+1$;

- if $A \equiv(B \rightarrow C) \rightarrow D$, then $d g(A)=d g(B \rightarrow C)+d g(C \rightarrow D)+1$.

It is easy to prove that $d g(A \rightarrow B)>d g(A)+d g(B)$. Given a swff $H \equiv \mathbf{T} A$ or $H \equiv \mathbf{F} A$, the degree of $H$ is $d g(H)=d g(A)$. For a finite set of swff's $S$ and a swff $H \in S$, the weight $\mathrm{wg}(H, S)$ of $H$ in $S$ is:

$$
\mathrm{wg}(H, S)= \begin{cases}0 & \text { if } H \equiv \mathbf{F}(A \rightarrow B) \text { and } \mathbf{T}((A \rightarrow B) \rightarrow C) \in S \\ d g(H) & \text { otherwise }\end{cases}
$$

The weight $\mathrm{wg}(S)$ of a finite set $S$ of swff's is $\mathrm{wg}(S)=\sum_{H \in S} \mathrm{wg}(H, S)$.

Lemma 2. Let $\Gamma$ be a finite and $\mathbf{T D}_{k}$-consistent set of swff's and let $\bar{\Gamma}$ be a node set of $\Gamma$.

(i) If $\left\{\Gamma_{0}, \ldots \Gamma_{u}\right\}$ is the sequence generating $\bar{\Gamma}, \operatorname{wg}\left(\Gamma_{i+1}\right) \leq \mathrm{wg}\left(\Gamma_{i}\right)$ for every $i \in\{0, \ldots, u-1\}$.

(ii) If $\Delta$ is a successor set of $\bar{\Gamma}, \operatorname{wg}(\Delta)<\operatorname{wg}(\bar{\Gamma})$.

Proof. The proof of Point (i) is trivial. Indeed, one can see that, if $R$ is any rule of Table 1 different from $\mathbf{T} \rightarrow \rightarrow, \Phi$ is the premise of $R$ and $\Psi$ is any component of the consequence of $R$, it holds that $\mathrm{wg}(\Psi)<\mathrm{wg}(\Phi)$. In the case of the $\mathbf{T} \rightarrow \rightarrow$-rule, $\operatorname{wg}(S \cup\{\mathbf{T} C\})<\operatorname{wg}(S \cup\{\mathbf{T}((A \rightarrow B) \rightarrow C)\})$, while $\operatorname{wg}(S \cup\{\mathbf{T}((A \rightarrow B) \rightarrow C)\})=\operatorname{wg}(S \cup\{\mathbf{F}(A \rightarrow B), \mathbf{T}((A \rightarrow B) \rightarrow C)\})$, since $\mathrm{wg}(\mathbf{F}(A \rightarrow B), S \cup\{\mathbf{T}((A \rightarrow B) \rightarrow C)\})=0$.

As for Point (ii), if $\Delta$ is a successor set corresponding to the rule $\mathbf{F} \vee$ the proof is trivial. Now, let us consider the case where $\Delta$ is a successor set of $\bar{\Gamma}$ corresponding to the rule $\mathrm{D}_{k}$. Then $\bar{\Gamma}=S \cup U \cup V$ where $U=\{H \in \bar{\Gamma} \mid H \equiv$ $\mathbf{F}(A \rightarrow B)\}, V=\{H \in \bar{\Gamma} \mid H \equiv \mathbf{T}((C \rightarrow D) \rightarrow E)\}$ and $S=\bar{\Gamma} \backslash(U \cup V)$. Let us suppose that

$$
\begin{aligned}
& U=\left\{\mathbf{F}\left(A_{1} \rightarrow B_{1}\right), \ldots, \mathbf{F}\left(A_{n} \rightarrow B_{n}\right)\right\} \\
& V=\left\{\mathbf{T}\left(\left(C_{1} \rightarrow D_{1}\right) \rightarrow E_{1}\right), \ldots, \mathbf{T}\left(\left(C_{m} \rightarrow D_{m}\right) \rightarrow E_{m}\right)\right\}
\end{aligned}
$$

We have two cases, according to the $\mathbf{T D}_{k}$-consistent hyperset used to build up the successor set $\Delta$.

Case 1: $\Delta$ is the only successor set of $\bar{\Gamma}$. In this case $\Delta=S_{c} \cup \Gamma_{i} \cup \Delta_{i}$ where $1 \leq i \leq n$ and

$$
\begin{aligned}
\Gamma_{i}= & U \backslash\left\{\mathbf{F}\left(A_{i} \rightarrow B_{i}\right)\right\} \cup\left\{\mathbf{T} A_{i}, \mathbf{F} B_{i}\right\} \\
\Delta_{i}= & \left\{\mathbf{T}\left(B_{i} \rightarrow E\right) \mid \mathbf{T}\left(\left(A_{i} \rightarrow B_{i}\right) \rightarrow E\right) \in V\right\} \\
& \cup\left\{\mathbf{T}((C \rightarrow D) \rightarrow E) \in V \mid C \rightarrow D \not \equiv A_{i} \rightarrow B_{i}\right\}
\end{aligned}
$$


If $\mathrm{wg}\left(\mathbf{F}\left(A_{i} \rightarrow B_{i}\right), \bar{\Gamma}\right)>0$ then there is no swff of the kind $\mathbf{T}\left(\left(A_{i} \rightarrow B_{i}\right) \rightarrow C\right)$ in $V$ and $\Delta_{i} \equiv V$. Since $d g\left(A_{i} \rightarrow B_{i}\right)>d g\left(A_{i}\right)+d g\left(B_{i}\right)$, it is easy to check that $\operatorname{wg}(\Delta)<\operatorname{wg}(\bar{\Gamma})$. On the other hand, if $\operatorname{wg}\left(\mathbf{F}\left(A_{i} \rightarrow B_{i}\right), \bar{\Gamma}\right)=0$, then $V$ contains at least a swff of the kind $\mathbf{T}\left(\left(A_{i} \rightarrow B_{i}\right) \rightarrow C\right)$ and we can write the sets $\bar{\Gamma}$ and $\Delta$ as follows:

$$
\begin{aligned}
& \bar{\Gamma}=\Xi \cup\left\{\mathbf{F}\left(A_{i} \rightarrow B_{i}\right)\right\} \cup\left\{\mathbf{T}\left(\left(A_{i} \rightarrow B_{i}\right) \rightarrow C_{1}\right), \ldots, \mathbf{T}\left(\left(A_{i} \rightarrow B_{i}\right) \rightarrow C_{q}\right)\right\} \\
& \Delta=\Xi_{c} \cup\left\{\mathbf{T} A_{i}, \mathbf{F} B_{i}\right\} \cup\left\{\mathbf{T}\left(B_{i} \rightarrow C_{1}\right), \ldots, \mathbf{T}\left(B_{i} \rightarrow C_{q}\right)\right\}
\end{aligned}
$$

where $q \geq 1$ and $\left\{\mathbf{T}\left(\left(A_{i} \rightarrow B_{i}\right) \rightarrow C_{1}\right), \ldots, \mathbf{T}\left(\left(A_{i} \rightarrow B_{i}\right) \rightarrow C_{q}\right)\right\}$ is the set of all the $\mathbf{T}$-signed implicative formulas occurring in $\bar{\Gamma}$ having $A_{i} \rightarrow B_{i}$ as antecedent. Now, given two sets of swff's $\Theta$ and $\Lambda$, let us denote with $\operatorname{wg}_{\Theta}(\Lambda)=$ $\sum_{H \in \Lambda} \operatorname{wg}(H, \Theta)$. It is easy to check that:

$$
\begin{aligned}
& \operatorname{wg}(\bar{\Gamma})=\operatorname{wg}_{\bar{\Gamma}}(\Xi)+d g\left(\left(A_{i} \rightarrow B_{i}\right) \rightarrow C_{1}\right)+\cdots+d g\left(\left(A_{i} \rightarrow B_{i}\right) \rightarrow C_{q}\right) \\
& \operatorname{wg}(\Delta) \leq \operatorname{wg}_{\Delta}\left(\Xi_{c}\right)+d g\left(A_{i}\right)+d g\left(B_{i}\right)+d g\left(B_{i} \rightarrow C_{1}\right)+\cdots+d g\left(B_{i} \rightarrow C_{q}\right)
\end{aligned}
$$

Since $\operatorname{wg}_{\Delta}\left(\Xi_{c}\right) \leq \operatorname{wg}_{\bar{\Gamma}}(\Xi), d g\left(A_{i}\right)+d g\left(B_{i}\right)+d g\left(B_{i} \rightarrow C_{1}\right)<d g\left(\left(A_{i} \rightarrow B_{i}\right) \rightarrow\right.$ $\left.C_{1}\right)$ and $d g\left(B_{i} \rightarrow C_{j}\right)<d g\left(\left(A_{i} \rightarrow B_{i}\right) \rightarrow C_{j}\right)$ for every $j \in\{2, \ldots, q\}$, it follows that $\operatorname{wg}(\Delta)<\operatorname{wg}(\bar{\Gamma})$.

Case 2: $\bar{\Gamma}$ has at least two successor sets. In this case, following the definition of $\mathrm{D}_{k}$, we can write $\Delta=S_{c} \cup U_{1} \cup \bar{V}_{1} \cup V_{2}$, where $U_{1} \subset U$ and $U_{1} \neq \emptyset$ and

$$
\begin{aligned}
\bar{V}_{1} & =\left\{\mathbf{T} E \mid \mathbf{T}((C \rightarrow D) \rightarrow E) \in V \text { and } \mathbf{F}(C \rightarrow D) \notin U_{1}\right\} \\
V_{2} & =\left\{\mathbf{T}((C \rightarrow D) \rightarrow E) \mid \mathbf{T}((C \rightarrow D) \rightarrow E) \in V \text { and } \mathbf{F}(C \rightarrow D) \in U_{1}\right\}
\end{aligned}
$$

On the other hand, we can write $\bar{\Gamma}$ as $S \cup U_{1} \cup U_{2} \cup V_{1} \cup V_{2}$ where $U_{2}=U \backslash U_{1}$ and $V_{1}=\left\{\mathbf{T}((C \rightarrow D) \rightarrow E) \in V \mid \mathbf{F}(C \rightarrow D) \notin U_{1}\right\}$ (we recall that $U_{1} \neq \emptyset$ and $\left.U_{2} \neq \emptyset\right)$. We notice that $\operatorname{wg}_{\Delta}\left(S_{c}\right) \leq \operatorname{wg}_{\bar{\Gamma}}(S)$. Moreover, if $\operatorname{wg}(\mathbf{F}(A \rightarrow B), \bar{\Gamma})=0$, where $\mathbf{F}(A \rightarrow B) \in U_{1}$, then there exists $C$ such that $\mathbf{T}((A \rightarrow B) \rightarrow C) \in V_{2}$, hence $\operatorname{wg}(\mathbf{F}(A \rightarrow B), \Delta)=0$ as well; it follows that $\operatorname{wg}_{\Delta}\left(U_{1}\right)=\operatorname{wg}_{\bar{\Gamma}}\left(U_{1}\right)$. Finally, if $V_{1} \neq \emptyset$, then $\overline{V_{1}} \neq \emptyset$ and $\operatorname{wg}_{\Delta}\left(\bar{V}_{1}\right)<\operatorname{wg}_{\bar{\Gamma}}\left(V_{1}\right)$. To prove that $\mathrm{wg}(\Delta)<$ $\mathrm{wg}(\bar{\Gamma})$ we have to consider two cases.

(i) There exists $\mathbf{F}(A \rightarrow B) \in U_{2}$ such that $\operatorname{wg}(\mathbf{F}(A \rightarrow B), \bar{\Gamma})>0$. Then $\operatorname{wg}_{\bar{\Gamma}}\left(U_{2}\right)>0$, therefore $\operatorname{wg}(\Delta)<\operatorname{wg}(\bar{\Gamma})$.

(ii) For all $\mathbf{F}(A \rightarrow B) \in U_{2}, \operatorname{wg}(\mathbf{F}(A \rightarrow B), \bar{\Gamma})=0$. Since $U_{2} \neq \emptyset$, there exist $\mathbf{F}(A \rightarrow B) \in U_{2}$ and $\mathbf{T}((A \rightarrow B) \rightarrow C) \in V_{1}$, hence $V_{1} \neq \emptyset$ and $\operatorname{wg}_{\Delta}\left(\bar{V}_{1}\right)<\operatorname{wg}_{\bar{\Gamma}}\left(V_{1}\right)$. This implies that $\operatorname{wg}(\Delta)<\operatorname{wg}(\bar{\Gamma})$.

Now, we have all the elements needed to prove that $\underline{K}_{\mathbf{D}}(S)$ is a Kripke model of $\mathcal{T}_{k}$.

Lemma 3. Let $S$ be a finite and $\mathbf{T D}_{k}$-consistent set of swff's and let $\underline{K}_{\mathbf{D}}(S)=$ $\langle P, \leq, \Vdash\rangle$ be the structure defined above. Then $\underline{K}_{\mathbf{D}}(S)$ is a Kripke model of $\mathcal{T}_{k}$. 
Proof. By construction of $\underline{K}_{\mathbf{D}}(S)$ it is easy to check that $\langle P, \leq\rangle$ is a poset; moreover, since $\mathbf{T} p \in \bar{\Gamma}$ implies that $\mathbf{T} p$ belongs to every successor set of $\bar{\Gamma}$, the forcing relation $\Vdash$ is well defined. Hence $\underline{K}_{\mathbf{D}}(S)$ is a Kripke model and, always by construction, every element of $\langle P, \leq\rangle$ has $k$ immediate successors at most. To conclude the proof we only have to show that $\langle P, \leq\rangle$ is finite. Let us assume that $\langle P, \leq\rangle$ is not finite; then, since $\langle P, \leq\rangle$ is a finite branching tree, there is an infinite chain $\bar{\Gamma}_{0}<\bar{\Gamma}_{1}<\ldots$ in $\langle P, \leq\rangle$. Since every $\bar{\Gamma}_{i}$ is finite and $\mathbf{T D}_{k}$-consistent, by the above lemma $\operatorname{wg}\left(\bar{\Gamma}_{0}\right)>\operatorname{wg}\left(\bar{\Gamma}_{1}\right)>\ldots$, which leads to a contradiction. Hence $\langle P, \leq\rangle$ is finite and this concludes the proof.

Lemma 4. Let $S$ be a finite and $\mathbf{T D}_{k}$-consistent set of swff's and let $\underline{K}_{\mathbf{D}}(S)=$ $\langle P, \leq, \Vdash\rangle$ be defined as above. Let $\bar{\Gamma} \in P$ and let $\left\{\Gamma_{0}, \ldots, \Gamma_{u}\right\}$ be the sequence generating $\bar{\Gamma}$. For every $i \in\{0, \ldots, u\}$ and every $H \in \Gamma_{i}, \bar{\Gamma} \triangleright H$ in $\underline{K}_{\mathbf{D}}(S)$.

Proof. The proof is by induction on $d g(H)$.

Basis: If $d g(H)=0$, then $H \equiv \mathcal{S} p$, with $p$ atom, and, by construction of $\underline{K}_{\mathbf{D}}(S)$, if $\mathcal{S} p \in \Gamma_{i}$ then $\mathcal{S} p \in \Gamma_{u}=\bar{\Gamma}$. If $\mathcal{S} \equiv \mathbf{T}$ then, by definition of forcing, $\bar{\Gamma} \triangleright \mathbf{T} p$; if $\mathcal{S} \equiv \mathbf{F}$ then, by consistency of $\bar{\Gamma}, \mathbf{T} p \notin \bar{\Gamma}$ and hence $\bar{\Gamma} \triangleright \mathbf{F} p$.

Step: Let us suppose that the assertion holds for every $H^{\prime}$ such that $d g\left(H^{\prime}\right)<$ $d g(H)$. The proof goes by cases on the structure of $H$. We give only few cases. If $H \equiv \mathbf{F}(A \rightarrow B)$ and $H \in \Gamma_{i}$, then, by construction, $H \in \bar{\Gamma}$. Since $\underline{K}_{\mathbf{D}}(S)$ is finite, there exists $\bar{\Delta} \in P$ such that $\bar{\Delta} \geq \bar{\Gamma}, H \in \bar{\Delta}$ and $\{\mathbf{T} A, \mathbf{F} B\}$ is included in a successor set $\Lambda$ of $\bar{\Delta}$. By induction hypothesis $\bar{\Lambda} \triangleright\{\mathbf{T} A, \mathbf{F} B\}$, thus $\bar{\Gamma} \triangleright \mathbf{F}(A \rightarrow B)$. If $H \equiv \mathbf{T}((A \rightarrow B) \rightarrow C)$ and $H \in \Gamma_{i}$, then, by construction, either $\mathbf{T} C \in \Gamma_{j}$ with $j>i$, or $\{\mathbf{T}((A \rightarrow B) \rightarrow C), \mathbf{F}(A \rightarrow B)\} \subseteq \bar{\Gamma}$. In the former case, by induction hypothesis, we immediately get $\bar{\Gamma} \triangleright \mathbf{T} \bar{C}$ and hence $\bar{\Gamma} \triangleright H$. In the latter case, let $\bar{\Lambda} \in P$ such that $\bar{\Lambda} \geq \bar{\Gamma}$; if $H \in \bar{\Lambda}$, then, by construction, $\mathbf{F}(A \rightarrow B) \in \bar{\Lambda}$, and by induction hypothesis $\bar{\Lambda} \nVdash A \rightarrow B$. If $H \notin \bar{\Lambda}$, then there exist $\bar{\Theta}_{1}, \bar{\Theta}_{2} \in P$ such that $\bar{\Gamma} \leq \bar{\Theta}_{1}<\bar{\Theta}_{2} \leq \bar{\Lambda}, H \in \bar{\Theta}_{1}, \mathbf{F}(A \rightarrow B) \in \bar{\Theta}_{1}$ and $H \notin \Theta_{2}$, with $\Theta_{2}$ a successor set of $\bar{\Theta}_{1}$ corresponding to the $\mathrm{D}_{k}$-rule. If $\mathbf{T} C \in \Theta_{2}$ then, by induction hypothesis, $\bar{\Theta}_{2} \triangleright \mathbf{T} C$, hence $\bar{\Lambda} \Vdash C$. On the other hand, if $\mathbf{T} A, \mathbf{T}(B \rightarrow C) \in \Theta_{2}$ then, by induction hypothesis, $\bar{\Theta}_{2} \triangleright \mathbf{T} A$ and $\bar{\Theta}_{2} \triangleright \mathbf{T}(B \rightarrow C)$, hence $\bar{\Lambda} \Vdash A$ and $\bar{\Lambda} \Vdash B \rightarrow C$. Therefore, if $\bar{\Lambda} \Vdash A \rightarrow B$, then $\bar{\Lambda} \Vdash C$. Thus we have proved that, for every $\bar{\Lambda} \in P$ such that $\bar{\Lambda} \geq \bar{\Gamma}$, if $\bar{\Lambda} \Vdash A \rightarrow B$, then $\bar{\Lambda} \Vdash C$ and this implies $\bar{\Gamma} \Vdash(A \rightarrow B) \rightarrow C$ and $\bar{\Gamma} \triangleright H$.

Theorem 3 (Completeness of $\mathbf{T D}_{k}$ ). If $A$ is valid in $\mathcal{T}_{k}$, then $A$ is provable in $\mathbf{T D}_{k}$.

Proof. Suppose that there is no closed $\mathbf{T D}_{k}$-proof table for $\{\mathbf{F} A\}$, then $\{\mathbf{F} A\}$ is a $\mathbf{T D}_{k}$-consistent set of swff's. By Lemma 4, $\mathbf{F} A$ is realizable in the model $\underline{K}_{\mathbf{D}}(\{\mathbf{F} A\}) \in \mathcal{T}_{k}$, hence $A$ is not valid in $\mathcal{T}_{k}$.

\section{Properties of $\mathrm{D}_{k}$}

First of all, we remark that in the calculus $\mathbf{T D}_{k}$ the swff's of the kind $\mathbf{F}(A \vee$ $B)$ are treated by two rules. This implies a non deterministic choice in the 
construction of a proof table. Of course, we can replace the rules $\mathbf{F} \vee_{1}$ and $\mathbf{F} \vee_{2}$ with the deterministic rule

$$
\frac{S, \mathbf{F}(A \vee B)}{S, \mathbf{F} A, \mathbf{F} B} \mathbf{F} \vee^{\prime}
$$

The resulting calculus $\mathbf{T} \mathbf{D}_{k}^{\prime}$ is trivially valid for $\mathbf{D}_{k}$. As for the completeness, we have to change the construction of the counter model $\underline{K}_{\mathbf{D}}(S)$ as follows: add the case

$(\mathcal{N} 9)$ If $H_{j} \equiv \mathbf{F}(A \vee B)$, then $\mathcal{U}\left(H_{j}, i\right)=\{\mathbf{F} A, \mathbf{F} B\}$

to the definition of node set and do not consider case $\mathcal{S} 1$ in the definition of successor set (thus, in this case the only successor sets are those corresponding to the $\mathrm{D}_{k}$-rule).

We have chosen to present the main calculus for $\mathbf{T D}_{k}$ with the rules $\mathbf{F} \vee_{1}$ and $\mathbf{F} \vee_{2}$ since they allow us to get an immediate and syntactical constructivity proof for $\mathbf{D}_{k}$.

Theorem 4. For every $k \geq 2$, if $A \vee B$ is provable in $\mathbf{T D}_{k}$ then either $A$ or $B$ is provable in $\mathbf{T D}_{k}$.

Proof. If $A \vee B$ is provable in $\mathbf{T} \mathbf{D}_{k}$ then there exists a closed $\mathbf{T} \mathbf{D}_{k}$-proof table $\tau$ for $\mathbf{F}(A \vee B)$. Since the first rule of $\tau$ is either $\mathbf{F} \vee_{1}$ or $\mathbf{F} \vee_{2}, \tau$ either contains a closed $\mathbf{T D}_{k}$-proof table for $\mathbf{F} A$ or a closed $\mathbf{T D}_{k}$-proof table for $\mathbf{F} B$.

Let us consider the rule characterizing the calculus $\mathbf{T D}_{k}$. Let $\Gamma=S \cup U \cup V$ where $U=\left\{\mathbf{F}\left(A_{1} \rightarrow B_{1}\right), \ldots, \mathbf{F}\left(A_{n} \rightarrow B_{n}\right)\right\}$ and $V=\left\{\mathbf{T}\left(\left(C_{1} \rightarrow D_{1}\right) \rightarrow\right.\right.$ $\left.\left.E_{1}\right), \ldots, \mathbf{T}\left(\left(C_{m} \rightarrow D_{m}\right) \rightarrow E_{m}\right)\right\}$. Since any complete $k$-sequence of $\Sigma_{U}^{k}$ is also a complete $(k+1)$-sequence of $\Sigma_{U}^{k+1}$, any component of the configuration $\mathcal{C}$ obtained by applying $\mathrm{D}_{k}$ to $S$ is also a component of the configuration $\mathcal{C}^{\prime}$ obtained by applying the rule $\mathrm{D}_{k+1}$ to $S$. This immediately implies that if $\{\mathbf{F} A\}$ has a closed $\mathbf{T} \mathbf{D}_{k+1}$-proof table then it also has a closed $\mathbf{T} \mathbf{D}_{k}$-proof table, therefore $\mathbf{D}_{k} \supseteq \mathbf{D}_{k+1}$. Moreover, since $\left(D_{k+1}\right)$ is not valid in the class of models $\mathcal{T}_{k}$, by the Completeness Theorem we get:

Theorem 5. For every $k \geq 2, \mathbf{D}_{k} \supset \mathbf{D}_{k+1}$.

We describe a procedure to decide $\mathbf{D}_{k}$ extracted from the completeness theorem for our tableau calculus. Here, for the sake of simplicity, we consider the tableau calculus $\mathbf{T D}_{k}^{\prime}$. Following the construction of the counter model $\underline{K}_{\mathbf{D}}(S)$, we can define the procedure $\Pi(\Gamma)$ of Table 2 that, taken as input a set $\Gamma$ of swff's, returns true if and only if $\Gamma$ is $\mathbf{T D}_{k}^{\prime}$-consistent. The procedure $\operatorname{Apply}(\Gamma)$ called in $\Pi$ takes as input a set $\Gamma$ of swff's and returns a configuration $\mathcal{C}_{P}$ obtained by selecting a main set of swff's $P$ in $\Gamma$ (if it exists) and applying a rule of $\mathbf{T D}_{k}^{\prime}$ to $\Gamma$ considering $P$ as the main set of swff's. We remark that lines 1-12 implement the cases $\mathcal{N} 1-\mathcal{N} 8$ of Section 3 and case $\mathcal{N} 9$ above of the construction of the node set, while lines 13-15 implement the construction of the successor sets (case $\mathcal{S} 2$ of Section 3 ). From the completeness of the decision procedure we get: 
Table 2. The procedures $\Pi(\Gamma)$ and $\operatorname{Apply}(\Gamma)$

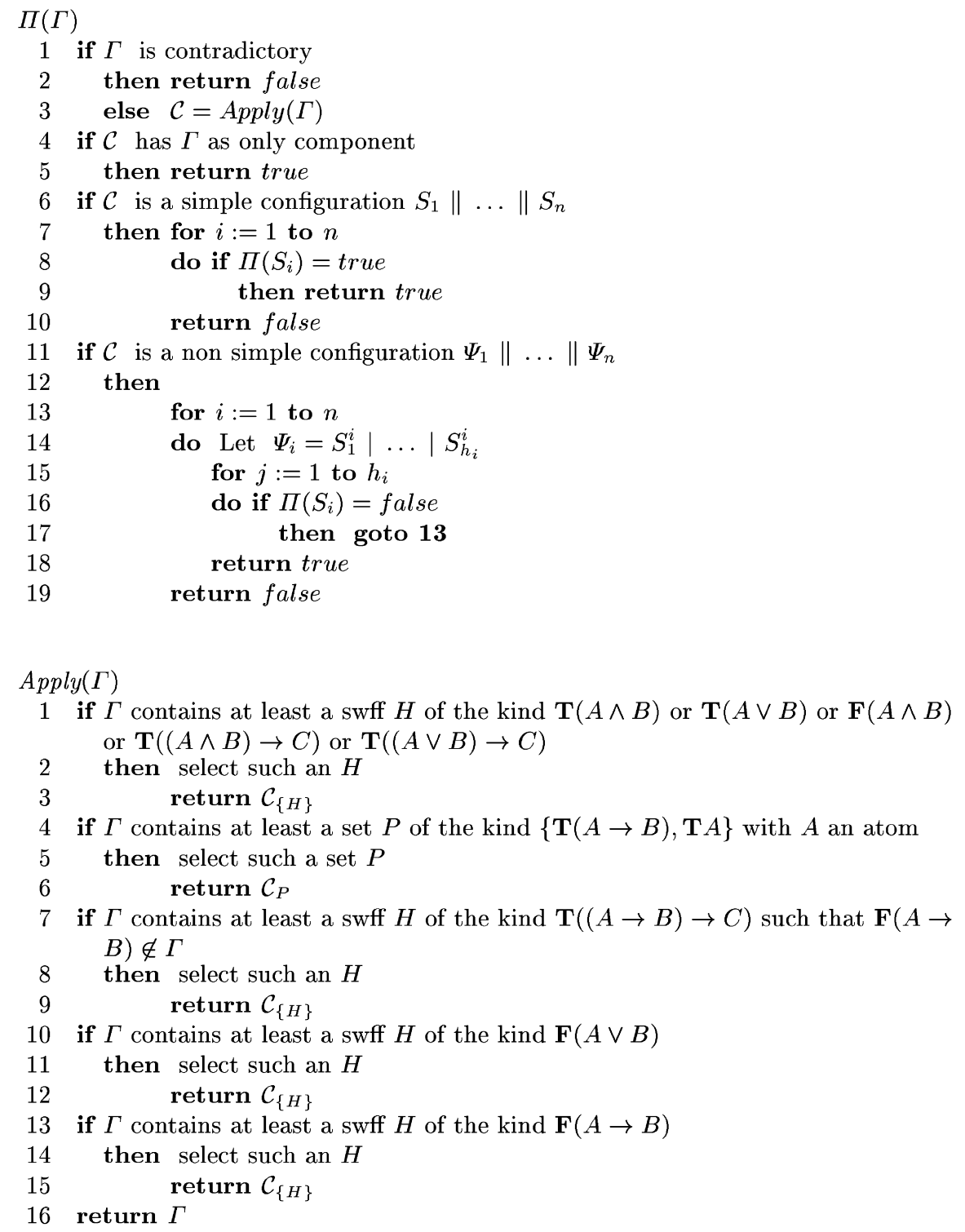

Theorem 6. For every $k \geq 2, \mathbf{D}_{k}$ is decidable.

To conclude the paper, we point out that the rule $\mathrm{D}_{k}$ is intrinsically inefficient, indeed the number of hypersets in the consequence of the rule is exponential in the number of $\mathbf{F}$-signed implicative formulas occurring in the premise. Despite 
this, our decision procedure is more efficient than the one based on generalized tableau given in 2], where in the proof one has to introduce a super-exponential number of instances of the axiom shema $\left(D_{k}\right)$.

\section{References}

1. A. Avellone, M. Ferrari, and P. Miglioli. Duplication-free tableau calculi and related cut-free sequent calculi for the interpolable propositional intermediate logics. Logic Journal of the IGPL, 7(4):447-480, 1999.

2. A. Avellone, P. Miglioli, U. Moscato, and M. Ornaghi. Generalized tableau systems for intermediate propositional logics. In D. Galmiche, editor, Proceedings of the 6th International Conference on Automated Reasoning with Analytic Tableaux and Related Methods: Tableaux '97, volume 1227 of LNAI, pages 43-61. Springer-Verlag, 1997.

3. A. Avron. Hypersequents, logical consequence and intermediate logics for concurrency. Annals for Mathematics and Artificial Intelligence, 4:225-248, 1991.

4. M. Baaz and C. G. Fermüller. Analytic calculi for projective logics. Lecture Notes in Computer Science, 1617:36-50, 1999.

5. A. Chagrov and M. Zakharyaschev. Modal Logic. Oxford University Press, 1997.

6. A. Ciabattoni and M. Ferrari. Hypertableau and path-hypertableau calculi for some families of intermediate logics. In R. Dyckhoff, editor, TABLEAUX 2000, Automated Reasoning with Analytic Tableaux and Related Methods, volume 1947 of $L N A I$, pages 160-174. Springer-Verlag, 2000.

7. A. Ciabattoni and M. Ferrari. Hypersequent calculi for some intermediate logics with bounded Kripke models. Journal of Logic and Computation, 11(2):283-294, 2001.

8. R. Dyckhoff. A deterministic terminating sequent calculus for Gödel-Dummett logic. Logic Journal of the IGPL, 7(3):319-326, 1999.

9. M. Ferrari and P. Miglioli. Counting the maximal intermediate constructive logics. Journal of Symbolic Logic, 58(4):1365-1401, 1993.

10. G. Fiorino. An $O(n \log n)$-SPACE decision procedure for the propositional Dummett Logic. Journal of Automated Reasoning, 27(3):297-311, 2001.

11. G. Fiorino. Space-efficient Decision Procedures for Three Interpolable Propositional Intermediate Logics. Journal of Logic and Computation, To appear.

12. M.C. Fitting. Intuitionistic Logic, Model Theory and Forcing. North-Holland, 1969.

13. D.M. Gabbay and D.H.J. De Jongh. A sequence of decidable finitely axiomatizable intermediate logics with the disjunction property. Journal of Symbolic Logic, 39:6778, 1974.

14. P. Hájek. Metamathematics of fuzzy logic. Kluwer, 1998.

15. D. Pearce. A new logical characterization of stable models and answer sets. In J. Dix, L.M. Pereira, and T. Przymusinski, editors, Non-Monotonic Extensions of Logic Programming, volume 1216 of LNAI, pages 57-70. Springer-Verlag, 1997.

16. D. Pearce. Stable inference as intuitionistic validity. Journal of Logic Programming, 38(1):79-91, 1999. 